\title{
Health Technology Assessment of Multiplex Reverse Transcriptase Polymerase Chain Reaction Screening for Translocations at Diagnosis in Acute Myeloid Leukaemia
}

Hans E Johnsen, ${ }^{1,2}$ Marianne H Hoffmann, ${ }^{2}$ Tobias W Klausen, ${ }^{2}$ Ulla H Davidsen, ${ }^{2}$ Olav J Bergmann, ${ }^{2}$ Martin Boegsted, ${ }^{1}$ Mette Nyegaard, ${ }^{1}$ Anne Bukh ${ }^{1}$ and Karen Dybkaer ${ }^{1}$

1. Department of Haematology, Aalborg Hospital, Aarhus University Hospital; 2. Herlev Hospital, University of Copenhagen

\begin{abstract}
It is predicted that gene diagnostics will become fundamental for future individualised treatment strategies in cancer patients following initial technological investigations and a step-by-step clinical validation. This report is a health technology assessment (HTA) of the use of a novel gene diagnostic assay in acute myeloid leukaemia (AML) with a focus on the technology, the patient, the organisation and economic aspects. In 202 patients with AML, a diagnostic bone marrow or a peripheral blood sample was available for gene analysis, identifying favourable risk translocations by a commercial (Hemavision ${ }^{\circledR}$ ) multiplex reverse transcription polymerase chain reaction (RT-PCR) Screening assay covering 28 fusion transcripts. The multiplex RT-PCR technology identified five fusion transcripts in five samples not identified by conventional cytogenetics and increased the number of patients identified as having a favourable prognosis from 16 to 21 ; one translocation identified by cytogenetics was not found by RT-PCR analysis. Using a prospective questionnaire analysis, a convincing 95\% (19/20) patient acceptance was documented for the diagnostic genetic analyses and the outcomes. However, patient understanding requires individual time and dialogue, in accordance with patient rights and modern individualised treatment strategies. The implementation of this strategy may benefit from already existing research laboratories forming a homogenous organisation using standardised technologies, which will make Denmark a high-quality partner in international clinical trials of new therapeutic strategies. The average economic cost per patient analysed using this technique is $€ 600$, which represents a modest $<1 \%$ increase in the total average cost of $€ 75,000$ for AML treatment. In conclusion, this HTA documented that the technology complements classic fluorescence in situ hybridisation (FISH) and provides additional information that can be used in response evaluation via already existing laboratories, but not without an increase in expense for health authorities. However, the increase in cost is limited compared with the costs of therapy and new drugs.
\end{abstract}

\section{Keywords}

Gene diagnostics, acute myeloid leukaemia, health technology assessment

Disclosure: The authors have no conflicts of interest to declare. The study was investigator-initiated and the authors paid for all of the kits used in the trial. Acknowledgements: The programme was supported in part by The Danish Centre for Evaluation and Health Technology Assessment (Grant 2001-4), the Novo Nordic Foundation (Senior Fellowship 2001-4) (HEJ) and the KE Jensen Foundation (Grant 2006 and 2010).

Received: 1 December 2009 Accepted: 18 February 2010 Citation: European Haematology, 2010;4:39-42

Correspondence: Hans E Johnsen, Professor, Clinical Haematology, Aalborg Hospital Science and Innovation Centre (AHSIC), Aarhus University Hospital,

Sdr Skovvej 15, DK-9000 Aalborg Denmark. E: haej@rn.dk

The opportunity for implementation of new technologies in healthcare is expanding with new findings in the areas of biotechnology and genetic research. A health technology assessment (HTA) can be used to answer questions related to implementation in clinical practice. However, the results of HTAs have limited impact on practical implementation decisions, especially in technologies introduced at an early stage of development.

Recently, many new genomic and genetic findings have been published in haematology, including multiplex reverse transcriptase polymerase chain reaction (RT-PCR)-based screening for translocations at diagnosis in acute myeloid leukaemia (AML). Given the heterogeneity of this disease, it is believed that improved outcome requires individualised treatment strategies at the time of diagnosis and therapeutic adjustment according to response. Insight into the variables of pathogenesis is rapidly progressing due to PCR-based and micro-array technology. ${ }^{1-3}$ However, the most important prognostic factors are still age and cytogenetics at diagnosis. ${ }^{4-11}$ Based on conventional chromosomal analysis, three groups of AML patients with relatively favourable, intermediate and adverse prognosis have been recognised and identified in the World Health Organization (WHO) classification of AML. ${ }^{12,13}$ As a consequence, the trend in current AML treatment protocols is to treat the favourable group with less intensive consolidation therapy and to offer experimental treatment to selected patients with intermediate and/or adverse prognosis.

Reliable identification of patients within the favourable risk group in particular is therefore highly desirable. While cytogenetic analysis reveals a broad spectrum of structural and numerical abnormalities, the analysis is in some cases hampered by the difficulty of obtaining sufficient quality of metaphases for analysis. As the cytogenetic basis of prognostic stratification is unsatisfactory in a fraction of AML patients, RT-PCR screening for reciprocal translocations seems an attractive complementary diagnostic modality. 
Table 1: Clinical Data for Adult Patients from Conclusive Multiplex Reverse Transcriptase Polymerase Chain Reaction

\begin{tabular}{|c|c|c|c|c|}
\hline & & $\begin{array}{l}\text { Adult Patients with } \\
\text { Multiplex RT-PCR } \\
\text { Performed }(n=191)\end{array}$ & $\begin{array}{l}\text { Adult Patients Diagnosed } \\
1 / 1 / 1992-31 / 12 / 2003 \\
(n=329)\end{array}$ & $\begin{array}{l}\text { Paediatric Patients with } \\
\text { Multiplex RT-PCR Performed } \\
(n=11)\end{array}$ \\
\hline \multirow[t]{3}{*}{ Age (years) } & Median & 66 & 67 & 4 \\
\hline & IQ range & $54-74$ & $56-75$ & $1-12$ \\
\hline & $\%<55$ & 28 & 24 & 100 \\
\hline Male/female ratio & & 1.17 & 1.23 & 1.75 \\
\hline \multirow{7}{*}{ FAB classification } & M1 & 35 & 61 & \\
\hline & M2 & 38 & 71 & 2 \\
\hline & M3 & 7 & 9 & \\
\hline & M4 & 37 & 62 & 5 \\
\hline & M5 & 14 & 14 & 1 \\
\hline & M6 & 1 & 6 & 2 \\
\hline & M7 & 3 & 9 & \\
\hline \multirow[t]{3}{*}{ \% blast cells in BM } & Median & 59 & 50 & 56 \\
\hline & Range & $1-100$ & $1-100$ & $11-90$ \\
\hline & IQ range & $32-81$ & $28-76$ & $15-80$ \\
\hline \multirow[t]{3}{*}{ WBCs in median peripheral blood } & Median & 14.6 & 10.0 & 18.6 \\
\hline & Range & $0.7-443.2$ & $0.2-443.2$ & $1.8-450.0$ \\
\hline & IQ range & $3.4-52.8$ & $2.3-42.3$ & $5.5-52.0$ \\
\hline CR rate* & & $66.0 \%(62 / 94)$ & $59.9 \%(91 / 152)$ & $90.9 \%(10 / 11)$ \\
\hline \multirow[t]{3}{*}{ Median survival in years } & Median & 0.4 & 0.4 & ND \\
\hline & Range & $0.0-9.1$ & $0.0-10.6$ & \\
\hline & IQ range* & $0.1-1.1$ & $0.1-1.1$ & \\
\hline Conclusive cytogenetics available & & $132(69 \%)$ & $188(57 \%)$ & $11(100 \%)$ \\
\hline
\end{tabular}

Clinical data for adult patients with conclusive multiplex reverse transcriptase polymerase chain reaction (RT-PCR) performed, for total number of adult patients diagnosed during the inclusion period and for paediatric patients with conclusive multiplex RT-PCR performed.

*Number of patients achieving complete response/number of patients with curative treatment intention.

$B M=$ bone marrow; $C R=$ complete response; $F A B=$ French-American-British; $I Q=$ interquartile; $N D=$ not declared; $W B C S=$ white blood cells.

The RT-PCR technology has the advantage of being independent of dividing cells. Furthermore, RT-PCR allows identification of the vast majority of patients with $t(8 ; 21)$, inv(16) or $t(15 ; 17)$, which defines the group of patients with relatively favourable treatment outcome. ${ }^{14-16}$ In addition, in fusion-gene-positive cases, a marker for minimal residual disease monitoring is identified.

In terms of the establishment of an overall consensus concerning gene diagnostics in haematology, we performed an HTA using retrospective and prospective data collected from an accessible regional clinical database and biobank.

\section{Materials and Method}

Trial Design and Approval

This HTA focused on aspects of the technology, the patient, the organisation and economic factors related to the introduction of a commercial multiplex RT-PCR screening assay covering 28 leukaemia fusion transcripts (HemaVision ${ }^{\circledR}$; DNA Technology, Aarhus, Denmark) identifying favourable risk translocations in AML. The programme was accepted by the local ethics committee in Copenhagen.

\section{Patients and Methods}

A total of 329 adult patients ( $>15$ years of age) were diagnosed with AML or chronic myeloid leukaemia (CML) blast crisis at a single institution during a 12-year period between 1 January 1992 and 31 December 2003. Clinical and biological information was collected from patient files for all adult patients with information available $(n=321,98 \%)$ to define the study group of 191 samples analysed (see Table 1). In addition, 11 paediatric patients with AML were included prospectively at the time of diagnosis. This patient material has been published previously.

RNA Extraction, Reverse Transcription and Multiplex Polymerase Chain Reaction

Total RNA was extracted using the QIAamp ${ }^{\circledR}$ RNA Blood Mini Kit (QIAGEN, Merck Euro lab A/S, Albertslund, Denmark) according to the manufacturer's recommendations. Analyses for balanced translocations were performed by multiplex RT-PCR analysis using the Hemavision screen test according to the manufacturer's recommendations. ${ }^{17}$

The commercially available multiplex RT-PCR assay detects 28 different chromosomal abnormalities by testing for the presence of more than 80 fusion transcript variants. Reverse transcription is performed with a mixture of translocation-specific primers. After complementary DNA (CDNA) synthesis, the PCR amplification is performed in two steps: a master PCR amplification followed by nested PCR, which screens for the presence of fusion transcripts, and a split-out PCR amplification followed by nested PCR, which identifies the specific fusion transcript(s).

\section{Cytogenetics}

Cytogenetic analysis was reported at diagnosis in 143 of the cases in which conclusive multiplex RT-PCR was performed. Clonal abnormalities were defined in accordance with the International System for Human Cytogenetic Nomenclature. 
Table 2: Agreement Between Multiplex Reverse Transcriptase Polymerase Chain Reaction (RT-PCR) and Cytogenetic Analysis Regarding Detection of Chromosomal Aberrations Included in the Multiplex RT-PCR Screen Test

\begin{tabular}{|c|c|c|c|}
\hline & $\begin{array}{l}\text { Fusion Transcript } \\
\text { Detected by Multiplex } \\
\text { RT-PCR Analysis }\end{array}$ & $\begin{array}{l}\text { No Fusion Transcript } \\
\text { Detected by Multiplex } \\
\text { RT-PCR Analysis }\end{array}$ & Total \\
\hline $\begin{array}{l}\text { Specific chromosomal abnormality } \\
\text { detected by cytogenetic analysis }\end{array}$ & 21 fusion transcripts in 16 samples & $\begin{array}{l}1 \text { fusion transcript in } 1 \text { sample } \\
\mathrm{t}(15 ; 17)(\mathrm{q} 22 ; \mathrm{q} 12)^{f}\end{array}$ & 1 fusion transcript in 17 samples \\
\hline $\begin{array}{l}\text { Specific chromosomal abnormality } \\
\text { not detected by cytogenetic analysis* }\end{array}$ & $\begin{array}{l}5 \text { fusion transcripts in } 5 \text { samples: } \\
\text { inv16(p13;q22) } \\
\text { t(8;21)(q22;q22) } \\
\text { t(8;21)(q22;q22) } \\
\text { t(15;17)(q21;q22) } \\
t(10 ; 11)(p 12 ; q 23)^{\mathrm{d}}\end{array}$ & 121 samples & 5 fusion transcripts in 126 samples \\
\hline Total & 26 fusion genes in 21 samples & 122 samples & 143 \\
\hline
\end{tabular}

The figures indicate the number of samples. The table includes samples in which both polymerase chain reaction (PCR) and cytogenetics were technically acceptable.

*Details of cytogenetic analysis results are as follows: a. Sample from adult. Karyotype: $50, X Y,+8,+9,+13,+14$. Number of mitoses examined not recorded. b. Sample from adult. Karyotype: $45, X,[15]$ add $7 q$ in two of 15 mitoses examined. c. Sample from child. Karyotype: 47,XY, del(8)(q12q22), del(9)(q12q22), der(11), add(11)(p14) del(11)(q23),+13,del(20)(p11) or q(11)[26]. d. Sample from child. Karyotype: 46,XX, add(5)(p14-15), del(15)(q15q22), add(17)(q11) [25]. Suspected abnormality of PML/RARA, spectral karyotyping: 46,XX, der(5)t(1;5)(p14-15;?), del(15)(q15;q22), both chromosome 17 normal. e. Sample from child. Karyotype: 46,XX [25]. f. Sample from adult, karyotype: 46,XX, t(15;17)(q22;q12) in 15 of 15 mitoses examined, verified by fluorescence in situ hybridisation (FISH). RT-PCR = reverse transcriptase polymerase chain reaction.

\section{Definition of End-points}

Overall survival was defined as the time from AML diagnosis to death. The criterion for complete remission (CR) was a normocellular bone marrow aspirate containing less than $5 \%$ blast cells and with normal maturation of other bone marrow cells, as evaluated by morphology and cytochemistry.

\section{Statistical Analysis}

Survival data analysis for adult patients was performed by construction of Kaplan-Meier life tables, with surviving patients being analysed on 31 December 2003. Eleven patients were lost to followup and were analysed at the date they were last known to be alive. Statistical analyses were performed by SPSS statistical software (SPSS Inc., Chicago, IL, version 121.0).

\section{Results}

Clinical and biological features are given in Table 1, including assignment to cytogenetic risk groups according to the results of the Medical Research Council Study 10 and 11 (MRC10 and MRC11) trials. ${ }^{3,4}$

\section{The Technology}

Multiplex RT-PCR analysis was conclusive in 191 adult and 11 paediatric cases. Twenty-six different fusion transcripts were detected in 21 patients (10\%), whereas no fusion transcript was detected in $90 \%$ of the patients. Cytogenetic and multiplex RT-PCR gave conflicting results in six patients regarding transcripts tested for by RT-PCR screening. Details of the agreement are given in Table 2. First, in one sample a $t(15 ; 17)$ was detected by cytogenetic analysis but not by RT-PCR. Second, in five cases inv16(p13;q22), t(8;21)(q22;q22), t(8;21)(q22;q22), t(15;17)(q21;q22) and $t(10 ; 11)(p 12 ; q 23)$ were detected by RT-PCR but not by cytogenetic analysis

\section{The Patient}

Patient attitudes to analysis for acquired genetic abnormalities were studied by a prospective survey questionnaire at diagnosis and following therapy in 20 consecutive patients. There was a convincing 95\% (19/20) acceptance of the gene diagnostic assay for acquired cancer genes to be used for later individual prediction regarding drug effect and prognosis.

\section{The Organisation}

Based on a survey within the Danish Multidisciplinary Cancer Groups, it was agreed that already existing research laboratories should be involved in implementaion, including registration into clinical databases and national biobanks. A homogenous organisation and standardised technologies will make Denmark a high-quality partner in international clinical trials of new strategies.

\section{The Economics}

The economic consequences of this treatment were analysed by retrospective and prospective cost analysis and calculated expenses for salary and running costs. In a prospective cost analysis of 79 samples, the cost per patient analysed was $€ 600$, against average total calculated treatment costs of $€ 75,000$ (range $€ 0-200,000, n=10$ ).

\section{Discussion}

Large clinical trials have demonstrated the prognostic value of diagnostic karyotyping, and the treatment strategy selected is now widely dependent on cytogenetic risk group assignment. ${ }^{4-7}$ In particular, safe identification of patients within the favourable risk group is highly desirable. However, cytogenetic analysis is in some cases hampered by the difficulty of obtaining metaphases of sufficient quality for analysis, making prognostic stratification unsatisfactory in a fraction of AML patients. Therefore, RT-PCR screening for reciprocal translocations seems an attractive complementary diagnostic modality to implement in clinical practice.

However, the point at which a new technology should be assessed remains a contentious issue. Broad clinical implementation and performing an HTA for policy decisions may be premature in the absence of prospective data on the actual benefits. However, if we wait to perform an HTA, it may be that worthwhile technology is withheld from the public health system. Coverage decisions usually have to be made at a time when the data on all of the relevant variables and adequate comparisons are not fully available from high-quality studies.

A patient's prognosis is usually based on clinical and pathological factors, such as age, leukaemia status, tumour burden and histological grading. However, these factors do not accurately predict the exact clinical behaviour, and therefore patients can be under- or, especially, over-treated. 
The value of multiplex RT-PCR has been validated in retrospective patient series. ${ }^{17,18}$ It is predicted that gene diagnostics will become fundamental for future individualised treatment strategies in cancer patients following initial technical investigations and a step-by-step clinical validation. However, it would take at least two to five years to bring the PCR-based technology into clinical practice by means of the usual strategy for prospective validation trials. Therefore, we decided to perform this HTA regarding the use of novel gene diagnostic assays in AML with a focus on the technology, the patient, the organisation and economic aspects.

In the technology assessment of this study, the HemaVision multiplex RT-PCR screening test was conclusive in all analyses performed prospectively. Retrospective analysis of seven samples that had been cryopreserved and stored for several years was inconclusive, probably due to RNA degradation. No difficulty in implementation of the assay in standard laboratory practice or in interpretation of test results was experienced. Surprisingly, the frequency of favourable-risk patients in this unselected population-based material was as low as $10 \%$, which is in contrast to data published by other authors indicating up to $45 \%$ with fusion genes detectable by multiplex RT-PCR. ${ }^{17,18}$ This difference may be due to sample selection bias.

Discrepancies between cytogenetic analysis and RT-PCR analysis suggesting the presence of cryptic abnormalities may be a consequence of suboptimal quality of the material obtained for cytogenetic analysis. However, in the cases described herein, there were no problems with the quality of the material examined by cytogenetic analysis. In fact, the risk of false-positive results is minimised by using the nested multiplex RT-PCR assay, in which positive analyses are confirmed in an additional nested split-out RT-PCR analysis. Although contamination cannot be completely ruled out, it seems unlikely, as to our knowledge no samples with detected abnormalities other than those described in this study were handled within the PCR area during the study period. Another theoretical explanation is that the fusion transcripts detected by RT-PCR may have been present in low cell numbers in nonmalignant cells and thus below the detection limit of the cytogenetic analysis. ${ }^{19}$

Our results support that for clinical prognostic evaluation, molecular methods such as RT-PCR or fluorescence in situ hybridisation (FISH) for detection of $t(8 ; 21), t(15 ; 17)$ and inv16 should be included in the diagnostic procedure to complement conventional cytogenetics for all AML patients, if curative treatment is intended. ${ }^{20}$
In the analysis of patient assessment, we dealt with individual aspects by prospective questionnaire and direct communication. In spite of the general ethical concerns related to analysis for hereditary genetic diseases, we were surprised how easy it was for patients with newly diagnosed AML to understand that the technology in question identified acquired genetic changes with an impact on disease prognosis and therapy. A convincing 95\% (19/20) patient acceptance rate was documented, but it must be stressed that this process requires time and dialogue, in accordance with patient rights and modern individualised treatment strategies.

The organisational assessment was assessed by the actual implementation of the new technology. The current organisation of gene diagnostic assays in haematology in Denmark is a consequence of historical development and compromises as part of a dynamic process. In the discussion of organisational matters, we found it very difficult to initiate a rational consensus discussion as each laboratory was focused on its own needs. Therefore, general implementation may benefit from already existing research laboratories forming a homogenous organisation of standardised technologies; this will make Denmark a high-quality partner in international clinical trials of new therapeutic strategies. During the economic assessment we used a simple cost minimisation analysis. In this type of analysis, it is sufficient to assess the actual costs. The average economic cost per patient analysed using this technique is $€ 600$, which represents a modest $<1 \%$ increase in the total average cost of $€ 75,000$ for AML treatment.

In conclusion, the technology is effective and complements classic cytogenetic methods. Technologically, evidence-based new gene diagnostic assays in haematology are in a position where they could be introduced tomorrow via already existing laboratories, but not without an increase in expenses for health authorities. However, the increase in cost is limited compared with the cost of new drugs introduced in cancer therapy.

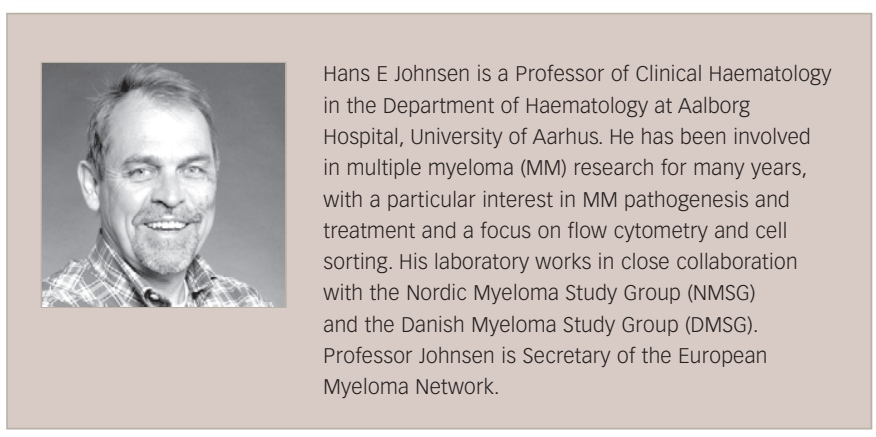

1. Hutchings Hoffmann $M$, Wirenfeldt Klausen $T_{\text {, Hasle }} \mathrm{H}_{\text {, }}$ et al., Haematologica, 2005;90(7):984-6.

2. Bullinger L, Dohner K, Bair E, et al., N Eng/ I Med, 2004;350:1605-16.

3. Valk PJ, Verhaak RG, Beijen MA, et al., N Eng/ J Med, 2004;350:1617-28.

4. Grimwade $D$, Walker $\mathrm{H}$, Oliver $\mathrm{F}$, et al., Blood, 1998;92:2322-33.

5. Grimwade D, Walker $\mathrm{H}$, Harrison $\mathrm{G}$, et al., Blood, 2001;98:1312-20

6. Slovak ML, Kopecky KJ, Cassileth PA, et al., Blood, 2000;96:4075-83.
7. Byrd JC, Mrozek K, Dodge RK, et al., Blood, 2002;100:4325-36

8. Smith M, Barnett M, Bassan R, et al., Blood Rev, 2004;18:115-36.

10. Grimwade D, Best Pract Res Clin Haematol, 2001;14:497-529.

11. Ferrara $F$, Lancet Oncol, 2004;5:443-50.

12. WHO histological classification of acute myeloid leukaemias. In: Swerdlow SH, Campo E, Harris NL, et al. (eds), IARC WHO Classification of Tumours, Volume 2 , 2001:75-107.

13. Vardiman JW, Harris NL, Brunning RD, Blood, 2002;100:2292-2302.
14. Rowe D, Cotterill SJ, Ross FM, et al., Br J Haematol, 2000;111:1051-6.

15. Mrozek K, Prior TW, Edwards C, et al., J Clin Oncol, 2001;19:2482-92.

16. Frohling S, Skelin S, Liebisch C, et al., J Clin Oncol, 2002;20:2480-85.

17. Pallisgaard N, Hokland P, Riishoj DC, et al., Blood, 1998:92:574-88.

18. Hokland P, Pallisgaard $N$, Semin Hematol, 2000;37:358-67

19. Strehl S, Konig M, Mann G, Haas OA, Blood, 2001;97:805-8.

20. Dohner H, Estey EH, Amadori S, et al., Blood, 2010:115(3):453-74 